\title{
Aspects of Holography and Rotating AdS Black Holes
}

\author{
David Berman * \\ Institute for Theoretical Physics \\ University of Groningen \\ Nijenborgh 4, 9747 AG Groningen, The Netherlands \\ E-mail: 'd.berman@phys. rug. nli'
}

ABSTRACT: A comparison is made between the thermodynamics of weakly and strongly coupled Yang-Mills with fixed angular momentum. The free energy of the strongly coupled Yang-Mills is calculated by using a dual supergravity description corresponding to a rotating black hole in an Anti de Sitter (AdS) background. All thermodynamic quantities are shown have the same ratio of $3 / 4$ (independent of angular momentum) between strong and weak coupling.

\section{Introduction}

The AdS/CFT correspondence [1] many insights into the connection between gauge theories and gravity. (See [ind for an extensive review of this vast subject.) In some sense the correspondence provides a duality map between large N Yang-Mills and IIB supergravity in an $A d S_{5} \times S^{5}$ background. The origin of this duality relation is from considering the physics of D3 branes from two perspectives. The world volume description, as can be derived from considering the D-brane as a submanifold on which strings can end, gives a Yang-Mills theory. The soliton description, whereby the the D-brane is seen as a solution in IIB supergravity provides, after taking appropriate limits, the alternative $A d S$ supergravity point of view. One may use this duality relation to calculate quantities in the YangMills theory at large ' $t$ Hooft coupling. In particular one may calculate thermodynamic quantities in the Yang-Mills theory from considering black holes in the AdS space-time [3]. Comparing these thermodynamic quantities with those calculated directly from the Yang-Mills theory at weak coupling, one sees that they correspond up to a factor of four thirds. (It should be stressed that there is no reason to expect them to match as typically entropies change as the coupling is

\footnotetext{
${ }^{*}$ This is work done in collaboration with M. Parikh
}

altered).

For nonrotating AdS black holes [4] modynamics has been described by thermal con-

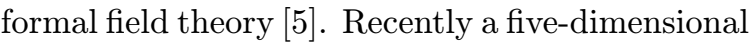
rotating black hole embedded in anti-de Sitter

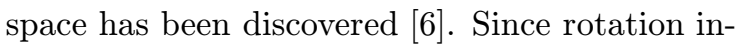
troduces an extra dimensionful parameter, the conformal field theory entropy is not so tightly constrained by the combination of extensivity and dimensional analysis; thus a comparison between thermodynamic quantities is much more nontrivial. Our purpose in this paper, as first reported

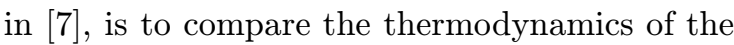
new rotating black hole with that of the dual conformal field theory in four dimensions. If one then assumes the AdS/CFT correspondence to be correct then the result may be interpreted as the comparison of thermodynamic quantites in the field theory at strong and weak coupling.

This correspondence has also been investigated in the case of rotating D branes by

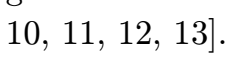

We begin by demonstrating the holographic [14 14, 15] nature of the duality for nonrotating black holes: the thermodynamics of a nonrotating black hole in anti-de Sitter space emerges from a thermal conformal field theory whose thermodynamic variables are read off from the boundary of the black hole spacetime. In the high temperature limit, the field theory calculation gives the cor- 
rect entropy of the Hawking-Page black hole up to a factor of $4 / 3$.

We then describe the new rotating Kerr-AdS black hole solution and show how its thermodynamic properties can be recovered from the dual field theory, in the high temperature limit. In that limit, the entropy, energy and angular momentum, compared with the statistical mechanics of the field theory, all agree with their gravitational counterparts, again up to a common factor of $4 / 3$.

\section{AdS/CFT Correspondence for Non- rotating Holes}

The five-dimensional Einstein-Hilbert action with a cosmological constant is given by

$$
I=-\frac{1}{16 \pi G_{5}} \int d^{5} x \sqrt{-g}\left(R+12 l^{2}\right)
$$

where $G_{5}$ is the five-dimensional Newton constant, $R$ is the Ricci scalar, the cosmological constant is $\Lambda=-6 l^{2}$, and we have neglected a surface term at infinity. Anti-de Sitter solutions derived from this action can be embedded in tendimensional IIB supergravity such that the supergravity background is of the form $A d S_{5} \times S^{5}$. The AdS/CFT correspondence then states that there is a dual conformal field theory in four dimensions from which one can extract the physics.

The line element of a "Schwarzschild" black hole in anti-de Sitter space [A-1] in five spacetime dimensions can be written as

$$
\begin{gathered}
d s^{2}=-\left(1-\frac{2 M G_{5}}{r^{2}}+r^{2} l^{2}\right) d t^{2} \\
+\left(1-\frac{2 M G_{5}}{r^{2}}+r^{2} l^{2}\right)^{-1} d r^{2}+r^{2} d \Omega_{3}^{2} .
\end{gathered}
$$

This solution has a horizon at $r=r_{+}$where

$$
r_{+}^{2}=\frac{1}{2 l^{2}}\left(-1+\sqrt{1+8 M G_{5} l^{2}}\right)
$$

The substitution $\tau=i t$ makes the metric positive definite and, by the usual removal of the conical singularity at $r_{+}$, yields a periodicity in $\tau$ of

$$
\beta=\frac{2 \pi r_{+}}{1+2 r_{+}^{2} l^{2}},
$$

which is identified with the inverse temperature of the black hole. The entropy is given by

$$
S=\frac{A}{4 G_{5}}=\frac{\pi^{2} r_{+}^{3}}{2 G_{5}},
$$

where $A$ is the "area" (that is 3 -volume) of the horizon.

We shall take the dual conformal field theory to be $\mathcal{N}=4, \mathrm{U}(\mathrm{N})$ super-Yang-Mills theory. But since it is only possible to do calculations in the weak coupling regime, we shall consider only the free field limit of Yang-Mills theory. Then, in the high-energy regime which dominates the state counting, the spectrum of free fields on a sphere is essentially that of blackbody radiation in flat space, with $8 N^{2}$ bosonic and $8 N^{2}$ fermionic degrees of freedom. The entropy is therefore

$$
S_{\mathrm{CFT}}=\frac{2}{3} \pi^{2} N^{2} V_{\mathrm{CFT}} T_{\mathrm{CFT}}^{3} .
$$

We would like to evaluate this "holographically", i.e. by substituting physical data taken from the boundary of the black hole spacetime. At fixed $r \equiv r_{0} \gg r_{+}$, the boundary line element tends to

$$
d s^{2} \rightarrow r_{0}^{2}\left[-l^{2} d t^{2}+d \Omega_{3}^{2}\right]
$$

The physical temperature at the boundary is consequently red-shifted to

$$
T_{\mathrm{CFT}}=\frac{T_{B H}}{\sqrt{-g_{t t}}}=\frac{T_{B H}}{l r_{0}},
$$

while the volume is

$$
V_{\mathrm{CFT}}=2 \pi^{2} r_{0}^{3}
$$

To obtain an expression for $N$, we invoke the AdS/CFT correspondence. Originating in the near horizon geometry of the D3-brane solution in IIB supergravity, the correspondence [i1], relates $N$ to the radius of $S^{5}$ and the cosmological constant:

$$
R_{S^{5}}^{2}=\sqrt{4 \pi g_{s} \alpha^{2} N}=\frac{1}{l^{2}} .
$$

Then, since

$$
(2 \pi)^{7} g_{s}^{2} \alpha^{\prime 4}=16 \pi G_{10}=16 \frac{\pi^{4}}{l^{5}} G_{5}
$$

we have

$$
N^{2}=\frac{\pi}{2 l^{3} G_{5}} .
$$


Substituting the expressions for $N, V_{\mathrm{CFT}}$ and $T_{\mathrm{CFT}}$ into Eq. $\left({ }_{2}^{2} \cdot 6^{i}\right)$, we obtain

$$
S_{\mathrm{CFT}}=\frac{1}{12} \frac{\pi^{2}}{l^{6} G_{5}}\left(\frac{1+2 r_{+}^{2} l^{2}}{r_{+}}\right)^{3}
$$

which, in the limit $r_{+} l \gg 1$, reduces to

$$
S_{\mathrm{CFT}}=\frac{2}{3} \frac{\pi^{2} r_{+}^{3}}{G_{5}}=\frac{4}{3} S_{\mathrm{BH}},
$$

in agreement with the black hole result, Eq. (2.2. but for a numerical factor of $4 / 3$.

Similarly, the red-shifted energy of the conformal field theory matches the black hole mass, modulo a coefficient. The mass above the anti-de Sitter background is

$$
M^{\prime}=\frac{3 \pi}{4} M
$$

This is the AdS equivalent of the ADM mass, or energy-at-infinity. The corresponding expression in the field theory is

$U_{\mathrm{CFT}}^{\infty}=\sqrt{-g_{t t}} \frac{\pi^{2}}{2} N^{2} V_{\mathrm{CFT}} T_{\mathrm{CFT}}^{4}=\frac{\pi}{2} r_{+}^{4} l^{2}=\frac{4}{3} M^{\prime}$

where $U_{\mathrm{CFT}}^{\infty}$ is the conformal field theory energy red-shifted to infinity, and we have again taken the $r_{+} l \gg 1$ limit. The $4 / 3$ discrepancy in Eqs. $(2.5)$ and $(\overline{1} . \overline{1} \overline{1})$ is construed to be an artifact of having calculated the gauge theory entropy in the free field limit rather than in the strong coupling limit required by the correspondence; intuitively, one expects the free energy to decrease when the coupling increases. The $4 / 3$ factor was first noticed in the context of D3-brane thermodynamics [3i]|. Our approach differs in that we take the idea of holography at face value, by explicitly reading physical data from the boundary of spacetime; nonetheless, Eq. (2.12i) refers to an underlying brane solution.

At this level, the correspondence only goes through in the $r_{+} l>>1$ limit. Note that, in terms of the conformal field theory $r_{+} l=T_{\mathrm{CFT}} r_{0}$. The limit we have taken means that $T_{\mathrm{CFT}} \gg$ $1 / r_{0}$, allowing us to neglect finite-size effects in the field theory which we have implicitly done in calculating the entropy in (2.6i).

\section{Five-Dimensional Rotating AdS Black Holes}

The general rotating black hole in five dimensions has two independent angular momenta. Here we consider the case of a rotating black hole with one angular momentum in an ambient AdS space. The line element is [6]

$$
\begin{aligned}
d s^{2}= & -\frac{\Delta}{\rho^{2}}\left(d t-\frac{a \sin ^{2} \theta}{\Xi_{a}} d \phi\right)^{2} \\
+ & \frac{\Delta_{\theta} \sin ^{2} \theta}{\rho^{2}}\left(a d t-\frac{\left(r^{2}+a^{2}\right)}{\Xi} d \phi\right)^{2} \\
& +\frac{\rho^{2}}{\Delta} d r^{2}+\frac{\rho^{2}}{\Delta_{\theta}} d \theta^{2}+r^{2} \cos ^{2} \theta d \psi^{2}
\end{aligned}
$$

where $0 \leq \phi, \psi \leq 2 \pi$ and $0 \leq \theta \leq \pi / 2$, and

$$
\begin{aligned}
\Delta & =\left(r^{2}+a^{2}\right)\left(1+r^{2} l^{2}\right)-2 M G_{5} \\
\Delta_{\theta} & =1-a^{2} l^{2} \cos ^{2} \theta \\
\rho^{2} & =r^{2}+a^{2} \cos ^{2} \theta \\
\Xi & =1-a^{2} l^{2}
\end{aligned}
$$

This solution is an anti-de Sitter space with curvature given by

$$
R_{a b}=-4 l^{2} g_{a b}
$$

The horizon is at

$r_{+}^{2}=\frac{1}{2 l^{2}}\left(-\left(1+a^{2} l^{2}\right)+\sqrt{\left(1+a^{2} l^{2}\right)^{2}+8 M G_{5} l^{2}}\right)$,

which can be inverted to give

$$
M G_{5}=\frac{1}{2}\left(r_{+}^{2}+a^{2}\right)\left(1+r_{+}^{2} l^{2}\right) .
$$

The entropy is one-fourth the "area" of the horizon:

$$
S=\frac{1}{2 G_{5}} \frac{\pi^{2}\left(r_{+}^{2}+a^{2}\right) r_{+}}{\left(1-a^{2} l^{2}\right)} .
$$

The entropy diverges in two different limits: $r_{+} \rightarrow$ $\infty$ and $a^{2} l^{2} \rightarrow 1$. The first of these descibes an infinite temperature and infinite radius black hole, while the second corresponds to "critical angular velocity", at which the Einstein universe at infinity has to rotate at the speed of light. The inverse Hawking temperature is

$$
\beta=\frac{2 \pi\left(r_{+}^{2}+a^{2}\right)}{r_{+}\left(1+a^{2} l^{2}+2 r_{+}^{2} l^{2}\right)} .
$$


The mass above the anti-de Sitter background is now

$$
M^{\prime}=\frac{3 \pi}{4 \Xi} M,
$$

the angular velocity at the horizon is

$$
\Omega_{H}=\frac{a \Xi}{r_{+}^{2}+a^{2}},
$$

and the angular momentum is defined as

$$
J_{\phi}=\frac{1}{16 \pi} \int_{S} \epsilon_{a b c d e} \nabla^{d} \psi^{e} d S^{a b c}=\frac{\pi M a}{2 \Xi^{2}},
$$

where $\psi^{a}=\left(\frac{\partial}{\partial \phi}\right)^{a}$ is the Killing vector conjugate to the angular momentum in the $\phi$ direction, and $\mathrm{S}$ is the boundary of a hypersurface normal to $\left(\frac{\partial}{\partial t}\right)^{a}$, with $d S^{a b c}$ being the volume element on $\mathrm{S}$.

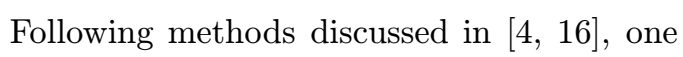
can derive a finite action for this solution from the regularized spacetime volume after an appropriate matching of hypersurfaces at large $r$. The result is

$$
I=\frac{\pi^{2}\left(r_{+}^{2}+a^{2}\right)^{2}\left(1-r_{+}^{2} l^{2}\right)}{4 G_{5} \Xi r_{+}\left(1+a^{2} l^{2}+2 r_{+}^{2} l^{2}\right)} .
$$

As noted in $\left[{ }^{1} \overline{6}_{-1}^{\prime}\right]$, the action changes sign at $r_{+} l=$ 1 , signalling the presence of a phase transition in the conformal field theory. For $r_{+} l>1$, the theory is in an unconfined phase and has a free energy proportional to $N^{2}$. One can also check that the action satisfies the thermodynamic relation

$$
S=\beta\left(M^{\prime}-J_{\phi} \Omega_{H}\right)-I .
$$

It is interesting to note that, by formally dividing both the free energy, $F=I / \beta$, and the mass by an arbitrary volume, one obtains an equation of state:

$$
p=\frac{1}{3} \frac{r_{+}^{2} l^{2}-1}{r_{+}^{2} l^{2}+1} \rho,
$$

where $p=-F / V$ is the pressure, and $\rho$ is the energy density. In the limit $r_{+} l \gg 1$ that we have been taking, this equation becomes

$$
p=\frac{1}{3} \rho,
$$

as is appropriate for the equation of state of a conformal theory. This suggests that if a conformal field theory is to reproduce the thermodynamic properties of this gravitational solution, it has to be in such a limit.

\section{The dual CFT description}

The gauge theory dual to supergravity on $A d S_{5} \times$ $S^{5}$ is $\mathcal{N}=4$ super Yang-Mills with gauge group $U(N)$ where $N$ tends to infinity [i].]. The action is

$$
\begin{array}{r}
S=\int d^{4} x \sqrt{g} \operatorname{Tr}\left(-\frac{1}{4 g^{2}} F^{2}+\frac{1}{2}(D \Phi)^{2}\right. \\
\left.+\frac{1}{12} R \Phi^{2}+\bar{\psi} \not D \psi\right) .
\end{array}
$$

All fields take values in the adjoint representation of $\mathrm{U}(\mathrm{N})$. The six scalars, $\Phi$, transform under $S O(6)$ R-symmetry, while the four Weyl fermions, $\psi$, transform under $S U(4)$, the spin cover of $S O(6)$. The scalars are conformally coupled; otherwise, all fields are massless. We shall again take the free field limit. The angular momentum operators can be computed from the relevant components of the stress energy tensor in spherical coordinates. This approach is to be contrasted with $\left[1 \overline{1} \bar{O}_{1}^{1}, \overline{1} \overline{1}_{1}^{1}, \overline{1} \overline{2}_{1}, \overline{1}, \overline{1} \overline{7}_{1}\right]$ in which generators of R-rotations are used corresponding to spinning D-branes.

The free energy of the gauge theory is given by

$$
\begin{array}{r}
F_{\mathrm{CFT}}=+T_{\mathrm{CFT}} \sum_{i} \eta_{i} \int_{0}^{\infty} d l_{i} \int d m_{i}^{\phi} \int d m_{i}^{\psi} \\
\ln \left(1-\eta_{i} e^{-\beta\left(\omega_{i}-m_{i}^{\phi} \Omega_{\phi}\right)}\right)(4
\end{array}
$$

where $i$ labels the particle species, $\eta=+1$ for bosons and -1 for fermions, $l_{i}$ is the quantum number associated with the total orbital angular momentum of the ith particle, and $m_{i}^{\phi(\psi)}$ is its angular momentum component in the $\phi(\psi)$ direction. Here $\Omega$ plays the role of a "voltage" while the "chemical potential" $m^{\phi} \Omega$ serves to constrain the total angular momentum of the system.

The free energy is easiest to evaluate in a corotating frame, which corresponds to the constanttime foliation choice of hypersurfaces orthogonal to $t^{a}$. Since, at constant $r=r_{0}$, the boundary has the metric

$$
\begin{aligned}
d s^{2} & =r_{0}^{2}\left[-l^{2} d t^{2}+\frac{2 a l^{2} \sin ^{2} \theta}{\Xi} d t d \phi+\frac{\sin ^{2} \theta}{\Xi} d \phi^{2}\right. \\
& \left.+\frac{d \theta^{2}}{\Delta_{\theta}}+\cos ^{2} \theta d \psi^{2}\right]
\end{aligned}
$$

the constant-time slices of the corotating frame have a spatial volume of

$$
V=\frac{2 \pi^{2} r_{0}^{3}}{1-a^{2} l^{2}} .
$$


The spectrum of a conformally coupled field on $S^{3}$ is essentially given by

$$
\omega_{l} \sim \frac{l}{r_{0}}
$$

where $l$ is the quantum number for total orbital angular momentum. Eq. (4.2i) can now be evaluated by making use of the identities

$$
\begin{aligned}
\int_{0}^{\infty} d x x^{n} \ln \left(1-e^{-x+c}\right) & =-\Gamma(n+1) \operatorname{Li}_{n+2}\left(e^{c}\right) \\
& =-\Gamma(n+1) \sum_{k=1}^{k=\infty} \frac{e^{k c}}{k^{n+2}} \\
\int d x x \operatorname{Li}_{2}\left(e^{-a x+c}\right) & =-\frac{1}{a^{2}}\left[a x \operatorname{Li}_{3}\left(e^{-a x+c}\right)\right. \\
& \left.+\operatorname{Li}_{4}\left(e^{-a x+c}\right)\right]
\end{aligned}
$$

where $\mathrm{Li}_{n}$ is the nth polylogarithmic function, defined by the sum above. The result is

$$
F_{\mathrm{CFT}}=-\frac{\pi^{4}}{24} \frac{r_{0}}{\frac{1}{r_{0}^{2}}-\Omega^{2}}\left(8 N^{2}\right) T_{\mathrm{CFT}}^{4},
$$

yielding an entropy of

$$
S_{\mathrm{CFT}}=\frac{2}{3} \frac{\pi^{5}}{l^{3} G_{5}} \frac{r_{0}^{3}}{1-\Omega^{2} r_{0}^{2}} T_{\mathrm{CFT}}^{3} .
$$

The physical temperature that enters the conformal field theory is

$$
T_{\mathrm{CFT}}=\frac{1}{l r_{0}} T_{\mathrm{BH}}
$$

Similarly, the angular velocity is scaled to

$$
\Omega_{\mathrm{CFT}}=\frac{a l^{2}}{l r_{0}} .
$$

Substituting Eqs. (4. and taking the high temperature limit as before, we have

$$
S_{\mathrm{CFT}}=\frac{2}{3 G_{5}} \frac{\pi^{2} r_{+}^{3}}{\left(1-a^{2} l^{2}\right)}=\frac{4}{3} S_{\mathrm{BH}}
$$

The inclusion of rotation evidently does not affect the ratio of the black hole and field theory entropies.

In the corotating frame, the free energy is simply of the form $N^{2} V T^{4}$, with the volume given by Eq. ('- $(\overline{4}-\overline{4})$. However, with respect to a nonrotating AdS space, the free energy takes a more complicated form since now the volume is simply $2 \pi^{2} r_{0}^{3}$. By keeping this volume and the temperature fixed, one may calculate the angular momentum of the system with respect to the nonrotating background:

$J_{\phi}^{\mathrm{CFT}}=-\left.\frac{\partial F}{\partial \Omega}\right|_{V, T_{\mathrm{CFT}}}=\frac{a r_{+}^{4} \pi\left(1+a^{2} l^{2}+2 r_{+}^{2} l^{2}\right)^{4}}{48 l^{6} \Xi^{2}\left(r_{+}^{2}+a^{2}\right)^{4}}$.

In the usual $r_{+} l \gg 1$ limit, we obtain

$$
J_{\phi}^{\mathrm{CFT}}=\frac{2 \pi M a}{3 \Xi^{2}}=\frac{4}{3} J_{\phi}^{\mathrm{BH}},
$$

so that the gauge theory angular momentum is proportional to the black hole angular momentum, Eq. (13.10 $)$, with a factor of $4 / 3$.

The black hole mass formula, Eq. (3.81), refers to the energy above the nonrotating anti-de Sitter background. We should therefore compare this quantity with the red-shifted energy in the conformal field theory. Here a slight subtlety enters. Since the statistical mechanical calculation gives the energy in the corotating frame, we must add the center-of-mass rotational energy before comparing with the black hole mass. Then we find that

$U_{\mathrm{CFT}}^{\infty}=\sqrt{-g_{t t}}\left(U_{\text {corotating }}+J_{\mathrm{CFT}} \Omega_{\mathrm{CFT}}\right)=\frac{4}{3} M^{\prime}$,

with $M^{\prime}$ given by Eq. $(\overline{3}-\overline{8})$, evaluated at high temperature. Using $U_{\mathrm{CFT}}^{\infty^{-}}=\sqrt{-g_{t t}} U_{\mathrm{CFT}}$ and previous expressions for thermodynamic quantities, one may check that the first law of thermodynamics is satisfied.

\section{Discussion}

There are several interesting aspects of these results. The first is that the same relative factor that appears in the entropy appears in the angular momentum and the energy. A priori, one has no reason to believe that the functional form of the free energy will be such as to guarantee this result (see, for example, $\left[10_{1}^{\prime}\right]$ ). The second is that the relative factor of $4 / 3$ in the entropy is unaffected by rotation. Indeed, one could expand the entropy of the rotating system in powers and inverse powers of the 't Hooft coupling. The cor- 
respondence implies that

$$
S_{\mathrm{CFT}}=\sum_{m} a_{m} \lambda^{m}=\sum_{n} b_{n}\left(\frac{1}{\sqrt{\lambda}}\right)^{n}=S_{\mathrm{BH}} .
$$

We may approximate the series on the gauge theory side as $a_{0}$ and on the gravity side as $b_{0}$. Then, generically, we would expect these coefficients to be functions of the dimensionless rotational parameter $\Xi$ so that $a_{0}(\Xi)=f(\Xi) b_{0}(\Xi)$ with $f(\Xi=1)=4 / 3$. Our somewhat unexpected result is that $f(\Xi)=4 / 3$ has, in fact, no dependence on $\Xi$. Ofcourse, in some sense the most fascinating aspect is that the ratio between strong and weak coupling is such a simple rational number. A futher understanding of this property of SYM would be very desirable.

Similar properties have been investigated with the incorporation of finite size effects by

\section{Acknowledgments}

We would like to thank José Barbón, Roberto Emparan, and Kostas Skenderis for helpful discussions. D. B. is supported by European Commission TMR programme ERBFMRX-CT96-0045. M. P. is supported by the Netherlands Organization for Scientific Research (NWO).

\section{References}

[1] J. Maldacena, "The Large N Limit of Superconformal field theories and supergravity," Adv. Theor. Math. Phys. 2 (1998) 231, hepth/9711200.

[2] O. Aharony, S. S. Gubser, J. Maldacena, H. Ooguri, and Y. Oz, "Large N Field Theories, String Theory and Gravity," hep-th/9905111.

[3] S. S. Gubser, I. R. Klebanov, and A. W. Peet, "Entropy and temperature of black 3-branes," Phys. Rev. D 54 (1996) 3915, hep-th/9602135.

[4] S. W. Hawking and D. N. Page, "Thermodynamics of Black Holes in Anti-de Sitter Space," Commun. Math. Phys. 88 (1983) 577, hepth/9811056.

[5] E. Witten, "Anti de Sitter Space and Holography," Adv. Theor. Math. Phys. 2 (1998) 253, hep-th/9802150.
[6] S. W. Hawking, C. J. Hunter, and M. M. TaylorRobinson, "Rotation and the AdS/CFT correspondence," Phys. Rev. D 59 (1999) 064005, hep-th/9811056.

[7] D. S. Berman, M. K. Parikh, "Holography and Rotating Black holes," Phys.Lett. B463 (1999) 168-173, hep-th/9907003.

[8] A. Chamblin, R. Emparan, C. V. Johnson, and R. C. Myers, "Charged AdS Black Holes and Catastrophic Holography," hep-th/9902170.

[9] A. Chamblin, R. Emparan, C. V. Johnson, and R. C. Myers, "Holography, Thermodynamics and Fluctuations of Charged AdS Black Holes," hep-th/9904197.

[10] S. S. Gubser, "Thermodynamics of spinning D3branes," Nucl. Phys. B551 (1999) 667, hepth/9810225.

[11] M. Cvetič and S. S. Gubser, "Thermodynamic Stability and Phases of General Spinning Branes," hep-th/9903132.

[12] M. Cvetič and S. S. Gubser, "Phases of Rcharged Black Holes, Spinning Branes and Strongly Coupled Gauge Theories," JHEP 9904 (1999) 024, hep-th/9902195.

[13] T. Harmark, N.A. Obers, "Thermodynamics of Spinning branes and their dual field theories", JHEP 0001 (2000) 008, hep-th/9910036.

[14] G. 't Hooft, "Dimensional Reduction in Quantum Gravity," University of Utrecht pre-print THU-93/26, gr-qc/9310026.

[15] L. Susskind, "The World as a Hologram," J. Math. Phys. 36 (1995) 6377, hep-th/9409089.

[16] E. Witten, "Anti-de Sitter Space, Thermal Phase Transition, And Confinement In Gauge Theories," Adv. Theor. Math. Phys. 2 (1998) 505, hep-th/9803131.

[17] M. Cvetič and F. Larsen, "Near Horizon Geometry of Rotating Black Holes in Five Dimensions," Nucl. Phys. B531 (1998) 239, hepth/9805097.

[18] S.W. Hawking, H.S. Reall, "Charged and rotating AdS black holes and their CFT duals," Phys.Rev. D61 (2000) 024014, hep-th/9908109.

[19] K. Landsteiner, E. Lopez, "The Thermodynamic Potentials of Kerr- AdS black holes and their CFT duals," JHEP 020 (1999) 9912, hepth/9911124. 